\title{
BAIKAL Experiment: main results obtained with the neutrino telescope NT200
}

\author{
V. Aynutdinov ${ }^{\text {a }}$, V. Balkanov ${ }^{\text {a }}$, I. Belolaptikov ${ }^{\text {d }}$, L. Bezrukov ${ }^{\text {a }}$, D. Borschov ${ }^{\text {a }}$, \\ N. Budnev ${ }^{b}$, I. Danilchenko a , Ya. Davidov ${ }^{\mathrm{a}}$, G. Domogatsky ${ }^{\mathrm{a}}, \mathrm{A}^{\mathrm{A}}$. Doroshenko ${ }^{\mathrm{a}}$, \\ A. Dyachok ${ }^{\text {b }}$, Zh.-A. Dzhilkibaev ${ }^{\text {a, }}{ }^{*}$, S. Fialkovsky $^{f}$, O. Gaponenko ${ }^{\text {a }}$, K. Golubkov ${ }^{\text {d }}$, \\ O. Gress ${ }^{\text {b }}$, T. Gress ${ }^{\text {b }}$, O. Grishin ${ }^{\text {b }}$, A. Klabukov ${ }^{\text {a }}$, A. Klimov ${ }^{\text {h }}$, S. Klimushin ${ }^{\text {a }}$, \\ A. Kochanov ${ }^{\text {b }}$, K. Konischev ${ }^{\text {d }}$, A. Koshechkin ${ }^{\text {a }}$, L. Kuzmichev ${ }^{c}$, V. Kulepov ${ }^{\mathrm{f}}$, \\ B. Lubsandorzhiev ${ }^{\mathrm{a}}$, S. Mikheyev ${ }^{\mathrm{a}}$, T. Mikolajski $^{\mathrm{e}}$, M. Milenin $^{\mathrm{f}}$, R. Mirgazov $^{\mathrm{b}}$, \\ E. Osipova ${ }^{\text {c }}$, A. Pavlov ${ }^{b}$, G. Pan'kov ${ }^{b}$, L. Pan'kov ${ }^{b}$, A. Panfilov ${ }^{a}$, D. Petukhov a \\ E. Pliskovsky ${ }^{\mathrm{d}}$, P. Pokhil ${ }^{\mathrm{a}}$, V. Poleschuk ${ }^{\mathrm{a}}$, E. Popova ${ }^{\mathrm{c}}$, V. Prosin ${ }^{\mathrm{c}}$, M. Rozanov $^{\mathrm{g}}$, \\ V. Rubtzov ${ }^{b}$, Yu. Semeney ${ }^{b}$, B. Shaibonov ${ }^{a}$, A. Shirokov ${ }^{c}$, Ch. Spiering $^{\mathrm{e}}$, \\ B. Tarashansky ${ }^{\text {b }}$, R. Vasiliev ${ }^{\mathrm{d}}$, R. Wischnewski ${ }^{\mathrm{e}}$, I. Yashin $^{\mathrm{c}}$, V. Zhukov ${ }^{\mathrm{a}}$ \\ ${ }^{\mathrm{a}}$ Institute for Nuclear Research, 60th October Anniversary pr. 7a, Moscow 117312, Russia \\ ${ }^{\mathrm{b}}$ Irkutsk State University, Irkutsk, Russia \\ c Skobeltsyn Institute of Nuclear Physics MSU, Moscow, Russia \\ d Joint Institute for Nuclear Research, Dubna, Russia \\ e DESY, Zeuthen, Germany \\ ${ }^{\mathrm{f}}$ Nizhni Novgorod State Technical University, Nizhni Novgorod, Russia \\ g St.Petersburg State Marine University, St.Petersburg, Russia \\ ${ }^{\mathrm{h}}$ Kurchatov Institute, Moscow, Russia
}

\begin{abstract}
The Baikal Neutrino Telescope NT200 takes data since April 1998. On April 9th, 2005, the 10 Mton scale detector NT200+ was put into operation in Lake Baikal. Selected results obtained during 1998-2002 with the neutrino telescope NT200 are presented.
\end{abstract}

Key words: Neutrino telescopes, Neutrino astronomy, UHE neutrinos, BAIKAL PACS: 95.55.Vj, 95.85.Ry, 96.40.Tv

\section{Introduction}

The Baikal Neutrino Telescope NT200 takes data since April 1998. On April 9th, 2005, the 10Mton scale detector NT200+ was put into operation in Lake Baikal. Description of site properties,

\footnotetext{
* Corresponding author.

Email address: djilkib@pcbai10.inr.ruhep.ru (Zh.-A. Dzhilkibaev).
}

detector configuration and performance have been described elsewhere $[1,2,3,4]$.

In this paper we present main results obtained from the analysis of data taken with the Baikal neutrino telescope NT200 between April 1998 and February 2003. 


\section{Selected results obtained with NT200}

\subsection{Atmospheric neutrinos}

The signature of charged current muon neutrino events is a muon crossing the detector from below. Muon track reconstruction algorithms and background rejection have been described elsewhere [5]. Compared to [5] the analysis of the 4-year sample (1038 days live time) was optimized for higher signal passing rate, and accepting a slightly higher contamination of $15-20 \%$ fake events [6]. A total of 372 upward going neutrino candidates were selected. From Monte-Carlo simulation a total of 385 atmospheric neutrino and background events are expected. The skyplot of these events is shown in Fig. 1.

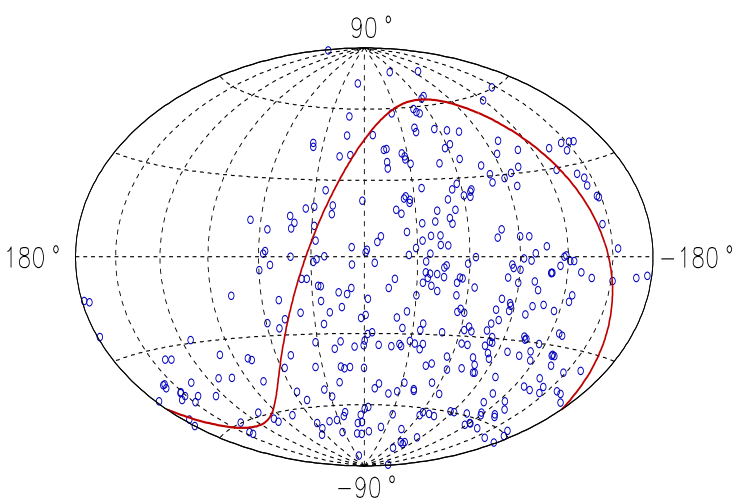

Fig. 1. Skyplot (galactic coordinates) of neutrino events for five years. The solid curve shows the equator.

\subsection{Search for Neutrinos from WIMP Annihilation}

The search for WIMPs with the Baikal neutrino telescope is based on a possible signal of nearly vertically upward going muons, exceeding the flux of atmospheric neutrinos. The method of event selection relies on the application of a series of cuts which are tailored to the response of the telescope to nearly vertically upward moving muons [7]. The applied cuts select muons with $-1<\cos (\theta)<-0.75$ and result in a detection area of about $1800 \mathrm{~m}^{2}$ for vertically upward going muons. The energy threshold for this analysis is $E_{\mathrm{thr}} \sim 10 \mathrm{GeV}$ i.e. significantly lower then for the analysis described

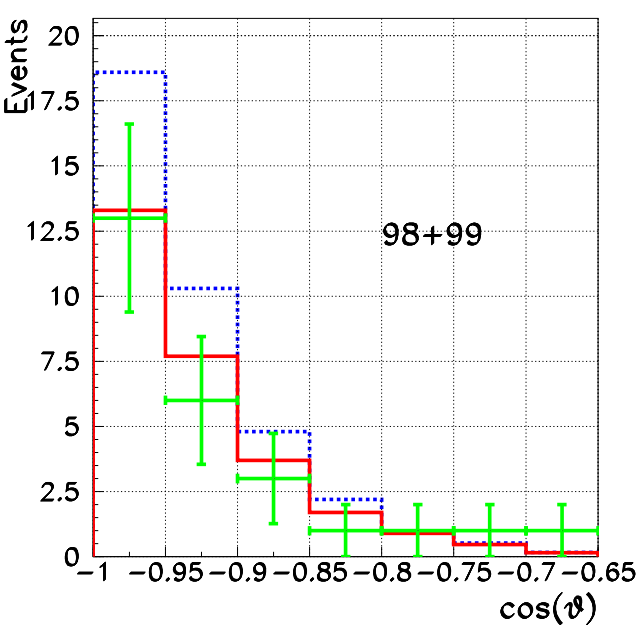

Fig. 2. Angular distributions of selected neutrino candidates as well as expected distributions in a case with and without oscillations (solid and dashed curves respectively).

in section $2.1\left(E_{\mathrm{thr}} \sim 15-20 \mathrm{GeV}\right)$. Therefore the effect of oscillations is stronger visible. We expect a muon event suppression of (25-30)\% due to neutrino oscillations assuming $\delta m^{2}=2.5 \cdot 10^{-3}$ $\mathrm{eV}^{2}$ with full mixing, $\theta_{m} \approx \pi / 4$.

From 502 days of effective data taking between April 1998 and February 2000, 24 events with $-1<$ $\cos (\theta)<-0.75$ have been selected as clear neutrino events. The angular distribution of these events as well as the MC - predicted distributions are shown in Fig. 2. For the MC simulations we used the Bartol96 atmospheric neutrino flux [8] without (dashed curve) and with (solid curve) oscillations. Within $1 \sigma$ statistical uncertainties the experimental angular distribution is consistent with the prediction including neutrino oscillations.

Regarding the 24 detected events as being induced by atmospheric neutrinos, one can derive an upper limit on the additional flux of muons from the center of the Earth due to annihilation of neutralinos - the favored candidate for cold dark matter. The $90 \%$ C.L. muon flux limits for six cones around the opposite zenith as well as muon flux limits for different neutralino masses obtained with NT200 $\left(E_{\text {thr }}>10 \mathrm{GeV}\right)$ in $1998 / 99$ are shown in Fig. 3 and Fig. 4, and compared to limits obtained by Baksan [9], MACRO [10], Super-Kamiokande [11] and AMANDA (from the hard neutralino annihilation channels) [12]. 


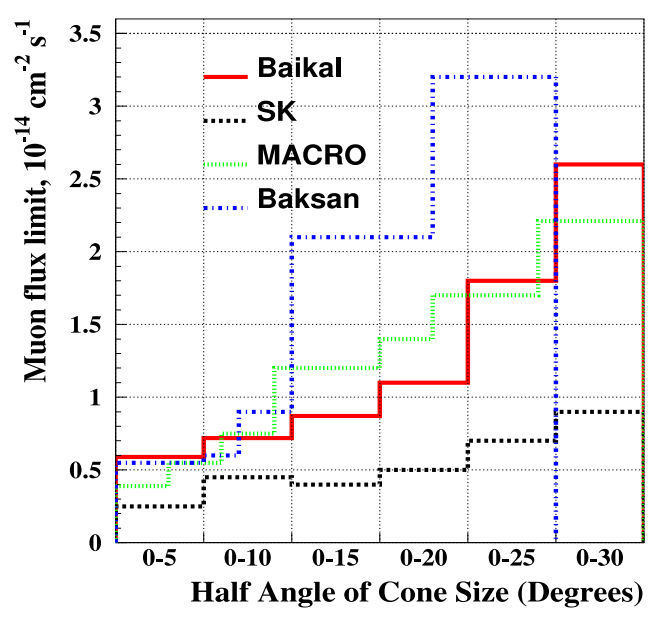

Fig. 3. Limits on the excess muon flux from the center of the Earth versus half-cone of the search angle.

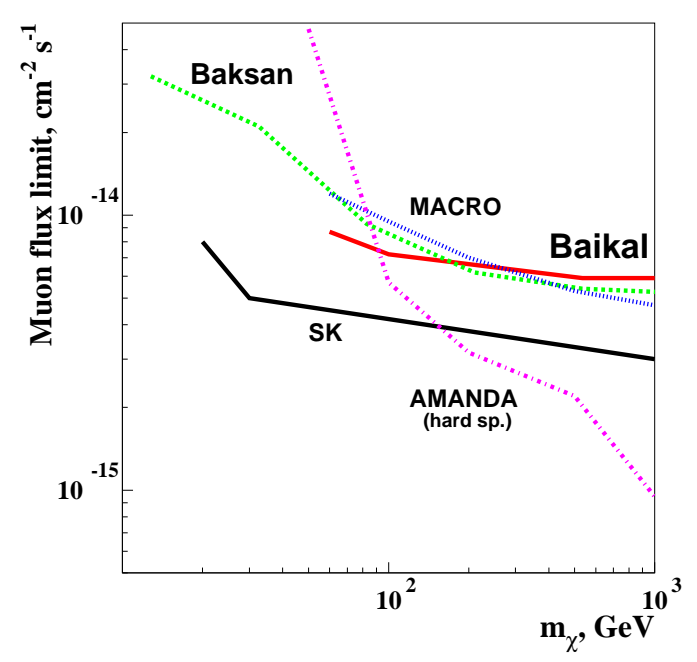

Fig. 4. Limits on the excess muon flux from the center of the Earth as a function of WIMP mass.

\subsection{A search for fast magnetic monopoles}

Fast magnetic monopoles with Dirac charge $g=$ $68.5 e$ are interesting objects to search for with deep underwater neutrino telescopes. The intensity of monopole Cherenkov radiation is $\approx 8300$ times higher than that of muons. Optical modules of the Baikal experiment can detect such an object from a distance up to hundred meters. The processing chain for fast monopoles starts with the selection of events with a high multiplicity of hit channels:
$N_{\text {hit }}>30$. In order to reduce the background from downward atmospheric muons we restrict ourself to monopoles coming from the lower hemisphere. For an upward going particle the times of hit channels increase with rising z-coordinates from bottom to top of the detector. To suppress downward moving particles, a cut on the value of the time- $\mathrm{z}^{-}$ correlation, $C_{t z}$, is applied:

$C_{t z}=\frac{\sum_{i=1}^{N_{h i t}}\left(t_{i}-\bar{t}\right)\left(z_{i}-\bar{z}\right)}{N_{h i t} \sigma_{t} \sigma_{z}}>0$

where $t_{i}$ and $z_{i}$ are time and z-coordinate of a fired channel, $\bar{t}$ and $\bar{z}$ are mean values for times and $\mathrm{z}^{-}$ coordinates of the event and $\sigma_{t}$ and $\sigma_{z}$ the rmserrors for time and $\mathrm{z}$-coordinates.

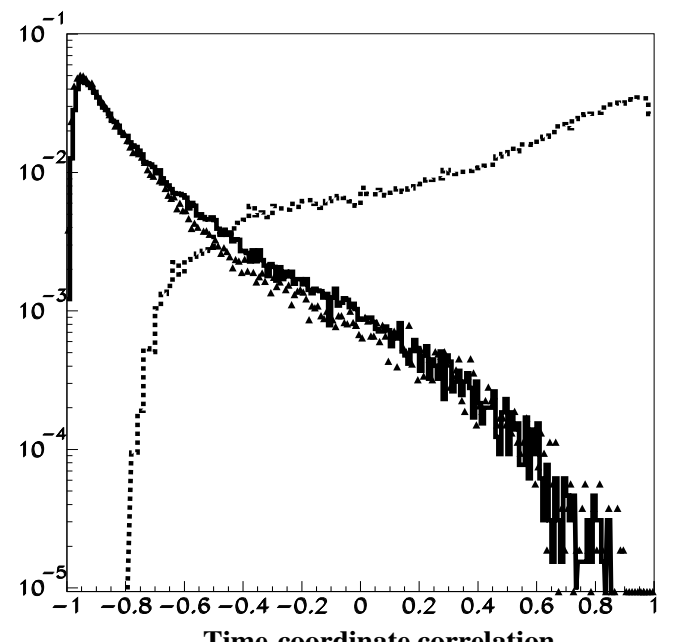

Fig. 5. $\mathrm{C}_{t z}$ distributions for experimental events (triangles), simulated atmospheric muon events (solid), and simulated upward moving relativistic magnetic monopoles (dotted); multiplicity cut $N_{h i t}>30$.

In Fig. 5 we compare the $C_{t z}$-distribution for experimental data (triangles), simulated atmospheric muon events (solid curve) with simulated upward moving monopole events (dotted curve).

Within 994 days of live time using in this analysis, about $3 \cdot 10^{8}$ events with $N_{\text {hit }}>4$ have been recorded, with 20943 of them satisfying cut 0 $\left(N_{\text {hit }}>30\right.$ and $\left.C_{t z}>0\right)$. For further background suppression (see [13] for details of the analysis) we use additional cuts, which essentially reject muon 
events and at the same time only slightly reduce the effective area for relativistic monopoles ${ }^{1}$ :

(i) $N_{h i t}>35$ and $C_{t z}>0.4 \div 0.6$

(ii) $\chi^{2}$ determined from reconstruction has to be smaller than 3

(iii) Reconstructed zenith angle $\theta>100 \mathrm{deg}$

(iv) Reconstructed track distance from NT200 center $R_{\text {rec }}>20 \div 25 \mathrm{~m}$.

No events from the experimental sample pass cuts 1-4. The acceptances $A_{\text {eff }}$ for monopoles with $\beta=1,0.9$ and 0.8 have been calculated for all NT200 operation configurations (various sets of operating channels). For the time periods included, $A_{\text {eff }}$ varies between $3 \cdot 10^{8}$ and $6 \cdot 10^{8} \mathrm{~cm}^{2} \mathrm{sr}$ (for $\beta=$ 1 ). From the non-observation of candidate events in NT200 and the earlier stage telescopes NT36 and NT96 [14], a combined upper limit on the flux of fast monopoles with $90 \%$ C.L. is obtained.

In Fig. 6 we compare this upper limit for an isotropic flux of fast monopoles obtained with the Baikal neutrino telescope to the limits from the underground experiments Ohya [15] and MACRO[16] and to the limit reported for the underice detector AMANDA[17]. The Baikal limit is currently the most stringent one.

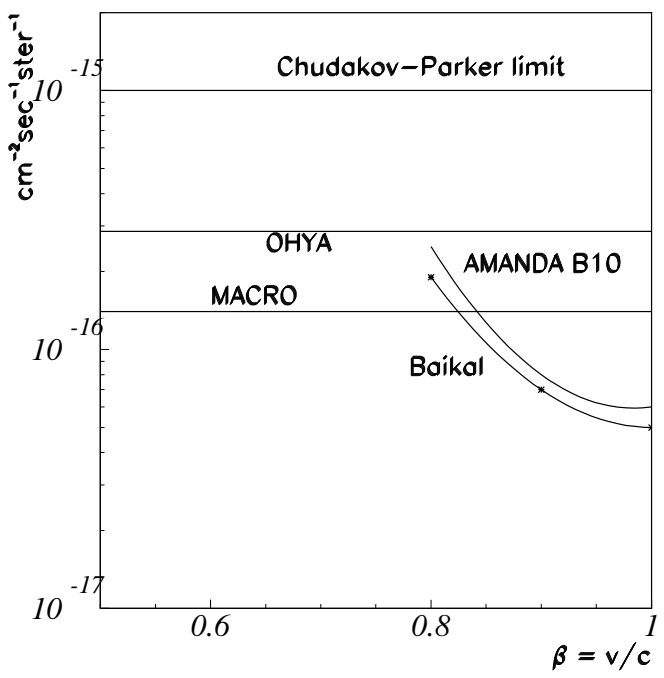

Fig. 6. Upper limits on the flux of fast monopoles obtained in this analysis (Baikal) and in other experiments.

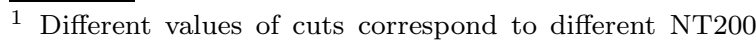
operation configurations.
}

\subsection{A search for extraterrestrial high-energy neutrinos}

The BAIKAL survey for high energy neutrinos searches for bright cascades produced at the neutrino interaction vertex in a large volume around the neutrino telescope [2]. We select events with high multiplicity of hit channels $N_{\text {hit }}$, corresponding to bright cascades. To separate high-energy neutrino events from background events a cut to select events with upward moving light signals has been developed. We define for each event $t_{\min }=$ $\min \left(t_{i}-t_{j}\right)$, where $t_{i}, t_{j}$ are the arrival times at channels $i, j$ on each string, and the minimum over all strings is calculated. Positive and negative values of $t_{\min }$ correspond to upward and downward propagation of light, respectively.

Within the 1038 days of the detector live time between April 1998 and February 2003, $3.45 \times$ $10^{8}$ events with $N_{\text {hit }} \geq 4$ have been recorded. For this analysis we used 22597 events with hit channel multiplicity $N_{\text {hit }}>15$ and $t_{\min }>-10$ ns. We conclude that data are consistent with simulated background for both $t_{\min }$ and $N_{\text {hit }}$ distributions. No statistically significant excess above the background from atmospheric muons has been observed. To maximize the sensitivity to a neutrino signal we introduce a cut in the $\left(t_{\min }, N_{\text {hit }}\right)$ phase space.

Table 1

Expected number of events $N_{\text {model }}$ and model rejection factors for models of astrophysical neutrino sources.

\begin{tabular}{lcc|c}
\hline & \multicolumn{2}{c|}{ BAIKAL } & AMANDA $[18,19,20]$ \\
\hline Model & $\nu_{e}+\nu_{\mu}+\nu_{\tau} n_{90 \%} / N_{\text {model }}$ & $n_{90 \%} / N_{\text {model }}$ \\
\hline $10^{-6} \times E^{-2}$ & 3.08 & 0.81 & 0.86 \\
SS Quasar [21] & 10.00 & 0.25 & 0.21 \\
SP u [22] & 40.18 & 0.062 & 0.054 \\
SP 1 [22] & 6.75 & 0.37 & 0.28 \\
P $p \gamma[23]$ & 2.19 & 1.14 & 1.99 \\
M $p p+p \gamma[24]$ & 0.86 & 2.86 & 1.19 \\
MPR [25] & 0.63 & 4.0 & 4.41 \\
SeSi [26] & 1.18 & 2.12 & - \\
\hline
\end{tabular}

Since no events have been observed which pass the final cuts upper limits on the diffuse flux of extraterrestrial neutrinos are calculated. For a $90 \%$ 

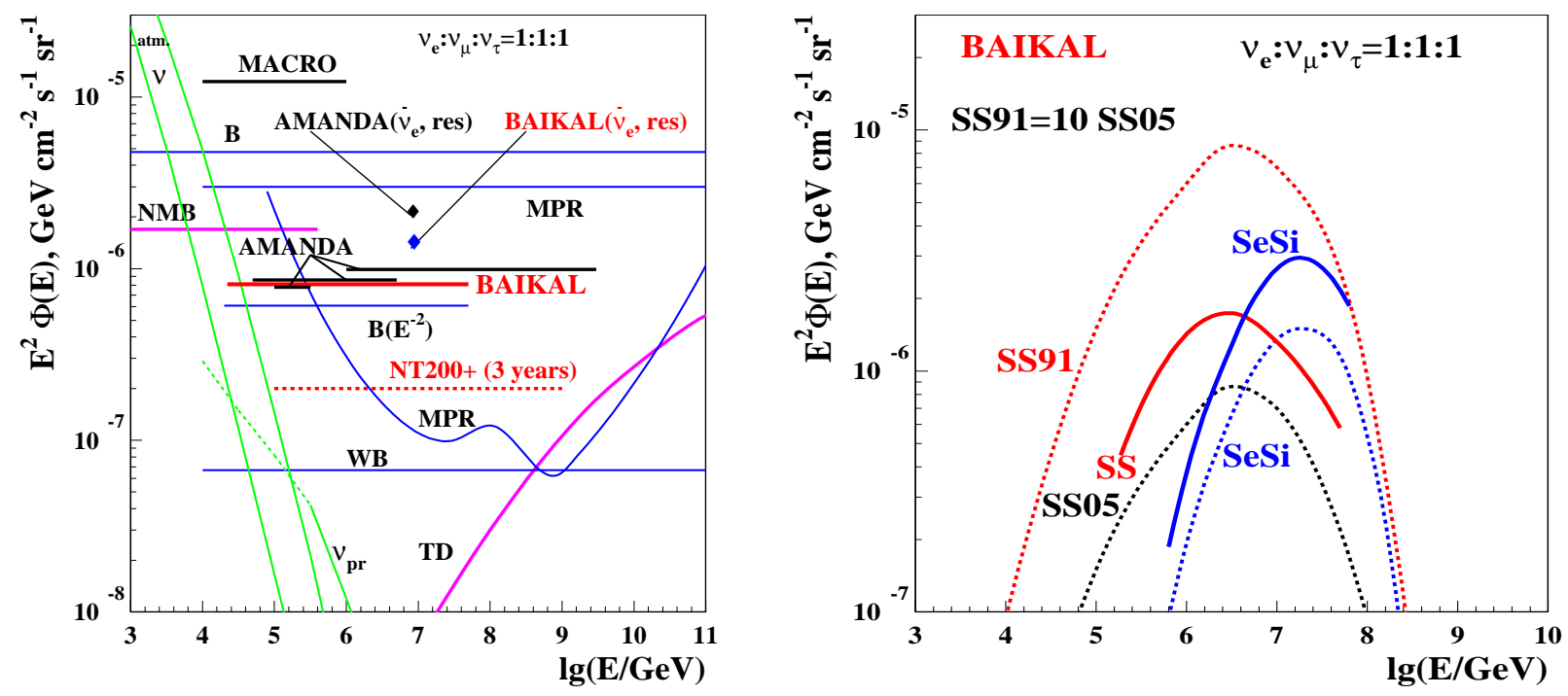

Fig. 7. Left panel: all-flavor neutrino flux predictions in different models of neutrino sources compared to experimental upper limits to $E^{-2}$ fluxes obtained by this analysis and other experiments (see text). Also shown is the sensitivity expected for 3 live years of the new telescope NT200+ [4,34]. Right panel: Baikal experimental limits compared to two model predictions. Dotted curves: predictions from model SS [21], SeSi [26] and SS05 [35]. Full curves: upper limits to spectra of the same shape. Model SS is excluded $(\mathrm{MRF}=0.25)$, model $\mathrm{SeSi}$ is not $(\mathrm{MRF}=2.12)$.

confidence level an upper limit on the number of signal events of $n_{90 \%}=2.5$ is obtained assuming an uncertainty in signal detection of $24 \%$ and a background of zero events.

A model of astrophysical neutrino sources, for which the total number of expected events, $N_{\text {model }}$, is large than $n_{90 \%}$, is ruled out at $90 \%$ CL. Table 1 represents event rates and model rejection factors (MRF) $n_{90 \%} / N_{\text {model }}$ for models of astrophysical neutrino sources obtained from our search, as well as model rejection factors obtained recently by the AMANDA collaboration $[18,19,20]$.

For an $E^{-2}$ behaviour of the neutrino spectrum and a flavor ratio $\nu_{e}: \nu_{\mu}: \nu_{\tau}=1: 1: 1$, the $90 \%$ C.L. upper limit on the neutrino flux of all flavors obtained with the Baikal neutrino telescope NT200 (1038 days) is:

$E^{2} \Phi<8.1 \times 10^{-7} \mathrm{~cm}^{-2} \mathrm{~s}^{-1} \mathrm{sr}^{-1} \mathrm{GeV}$.

For the resonant process with the resonant neutrino energy $E_{0}=6.3 \times 10^{6} \mathrm{GeV}$ the modelindependent limit on $\overline{\nu_{e}}$ is:

$$
\Phi_{\overline{\nu_{e}}}<3.3 \times 10^{-20} \mathrm{~cm}^{-2} \mathrm{~s}^{-1} \mathrm{sr}^{-1} \mathrm{GeV}^{-1} .
$$

Fig. 7 (left panel) shows our upper limit on the all flavor $E^{-2}$ diffuse flux (2) as well as the model independent limit on the resonant $\bar{\nu}_{e}$ flux (diamond) (3). Also shown are the limits obtained by AMANDA $[18,19,20]$ and MACRO [27], theoretical bounds obtained by Berezinsky (model independent (B) [28] and for an $E^{-2}$ shape of the neutrino spectrum $\left(\mathrm{B}\left(E^{-2}\right)\right)[29]$, by Waxman and Bahcall (WB) [30], by Mannheim et al.(MPR) [25], predictions for neutrino fluxes from topological defects (TD) [26], prediction on diffuse flux from AGNs according to Nellen et al. (NMB) [31], as well as the atmospheric conventional neutrino fluxes [32] from horizontal and vertical directions ( $(\nu)$ upper and lower curves, respectively) and atmospheric prompt neutrino fluxes $\left(\nu_{p r}\right)$ obtained by Volkova et al. [33]. The right panel of Fig. 7 shows our upper limits (solid curves) on diffuse fluxes from AGNs shaped according to the model of Stecker and Salamon (SS, SS05) [21,35] and of Semikoz and Sigl (SeSi) [26], according to Table 1. 


\section{Conclusion}

The Baikal neutrino telescope NT200 is taking data since April 1998. The upper limit obtained for a diffuse $\left(\nu_{e}+\nu_{\mu}+\nu_{\tau}\right)$ flux with $E^{-2}$ shape is $E^{2} \Phi=8.1 \times 10^{-7} \mathrm{~cm}^{-2} \mathrm{~s}^{-1} \mathrm{sr}^{-1} \mathrm{GeV}$. The limits on fast magnetic monopoles and on a $\overline{\nu_{e}}$ flux at the resonant energy $6.3 \times 10^{6} \mathrm{GeV}$ are presently the most stringent. To extend the search for diffuse extraterrestrial neutrinos with higher sensitivity, NT200 was significantly upgraded to NT200+, a detector with about 5 Mton enclosed volume, which takes data since April 2005 [4,34]. The three-year sensitivity of NT200+ to the all-flavor neutrino flux is approximately $2 \times 10^{-7} \mathrm{~cm}^{-2} \mathrm{~s}^{-1} \mathrm{sr}^{-1} \mathrm{GeV}$ for $E>10^{2} \mathrm{TeV}$ (shown in Fig. 7). In parallel with exploiting NT200+ we started research \& development activities towards a Gigaton Volume Detector in Lake Baikal.

AcknowledgementsThis work was supported by the Russian Ministry of Education and Science, the German Ministry of Education and Research and the Russian Fund of Basic Research (grants 05-0217476, 04-02-17289, 04-02-16171, 05-02-31021, 0502-16593), and by the Grant of the President of Russia NSh-1828.2003.2.

\section{References}

[1] V.Aynutdinov et al. Nucl. Phys. (Proc. Suppl.) B143 (2005) 335.

[2] V.Aynutdinov et al. Astropart. Phys. 25 (2006) 140.

[3] V.Aynutdinov et al. Proc. of V Int. Conf. on NonAccelerator New Physics June 7-10 Dubna Russia.

[4] V.Aynutdinov et al. "Status of the Baikal Neutrino Telescope NT200+", these Proceedings.

[5] V.Balkanov et al. Astropart. Phys. 12 (1999) 75.

[6] R.Wischnewski et al. Int. J. Mod. Phys. B20 (2005) 6932 .

[7] V. Balkanov et al. Nucl.Ph.(Proc.Sup.) B91 (2001) 438.
[8] V. Agrawal, T. Gaisser, P. Lipari \& T. Stanev Phys. Rev. D53 (1996) 1314.

[9] M.M.Boliev et al. Nucl. Phys. (Proc. Suppl.) 48 (1996) 83 ; O. Suvorova arXiv.org: hep-ph/9911415 (1999).

[10] M.Ambrosio et al. Phys. Rev. D60 (1999) 082002.

[11] S.Desai et al. Phys. Rev. D70 (2004) 083523, erratum ibid D70 (2004) 109901

[12] J. Ahrens et al. arXiv.org: astro-ph/0509330 (2005)

[13] V.Aynutdinov et al. Proc. 29th Int. Cosmic Ray Conf. August 3-10 Pune India (arXiv.org: astro-ph/0507713 (2005))

[14] I.Belolaptikov et. al. [Baikal collaboration], 26th ICRC, Salt Lake City, V.2 (1999) 340.

[15] S.Orito et. al., Phys.Rev.Lett. 66 (1991) 1951.

[16] M.Ambrosio et. al. [MACRO collaboration], arXiv.org: hep-ex/0200702p (2002).

[17] P.Niessen, C.Spiering, [AMANDA collaboration], 27th ICRC, Hamburg, V.4 (2001) 1496.

[18] M.Ackermann et al. Astropart. Phys. 22 (2005) 127, Astropart. Phys. 22 (2005) 339.

[19] M. Ackermann et al., Astropart. Phys. 22 (2005) 339.

[20] K.Muenich et al. Proc. 29th Int. Cosmic Ray Conf. August 3-10 (2005) Pune India, (arXiv.org: astro-ph/0509330 (2005)).

[21] F. Stecker and M. Salamon, Space Sci. Rev. 75 (1996) 341.

[22] A. Szabo and R. Protheroe, Proc. High Energy Neutrino Astrophy- sics, ed. V.J. Stenger et al., Honolulu, Hawaii (1992).

[23] R.J. Protheroe, arXiv.org:astro-ph /9612213.

[24] K. Mannheim, Astropart. Phys. 3 (1995) 295.

[25] K. Mannheim, R.J. Protheroe and J.P. Rachen, Phys. Rev. D63 (2001) 023003.

[26] D. Semikoz and G. Sigl, arXiv.org hep-ph/0309328

[27] M.Ambrosio et al. Nucl. Phys. (Proc. Suppl.) B110 (2002) 519

[28] V.Berezinsky et al. Astrophysics of Cosmic Rays (1990) Amsterdam: North Hollands.

[29] V.Berezinsky arXiv.org: astro-ph/0505220 (2005).

[30] E.Waxman and J.Bahcall Phys. Rev. D59 (1999) 023002.

[31] L.Nellen, K.Mannheim and P.Biermann Phys. Rev. D47 (1993) 5270.

[32] L.Volkova Yad. Fiz. 31 (1980) 1510.

[33] L.Volkova and G.T.Zatsepin Phys. Lett. B462 (1999) 211. 
[34] V. Aynutdinov, V. Balkanov et al., arXiv.org: astro-ph /0507715.

[35] F. Stecker arXiv.org: astro-ph /0510537. 